Vol. 4, Special Issue 2, January 2017

\title{
USB to USB Data Transfer using Raspberry Pi and ARM
}

\author{
Miss. Monika T. Shinde ${ }^{1}$, Mr. Ramchandra. K. Gurav ${ }^{2}$ \\ Student -Bachelor of Engineering, Electronics \& Telecommunication Engineering Department, Dr. Daulatrao Aher \\ College of Engineering, Karad, Maharashtra, India ${ }^{1}$ \\ Assistant Professor, Electronics \& Telecommunication Engineering Department, Dr. Daulatrao Aher College of \\ Engineering, Karad, Maharashtra, India ${ }^{2}$
}

\begin{abstract}
An importance of portability is well known to us so to achieve this, we are going to design a system which we can carry anywhere. Using this system we can not only transfer data but also we are able to see the transfer of the particular file which we are going to transfer by using graphical display. Hence the system to be designed will be more compact .In our project we are going transfer data between different USB devices using Raspberry pi and ARM7 without using any computer or laptop .As shown in block diagram four USB devices can be connected to Raspberry pi while one USB device can be connected to ARM 7.Out of these various devices one device can act as a master while others will act as slaves. After sending particular command to processor ,the processor will start fetching data from master USB device and ARM processor will wait from the signal from destination or slave USB.As soon as processor gets the signal from destination device data transfer operation get started.
\end{abstract}

Keyword: Raspberry Pi, ARM7, USB, Graphical display.

\section{INTRODUCTION}

As we discussed earlier importance as well as need of portable devices is known to every one of us.

It is very easy to find an USB device along with its application around us everywhere.

Carrying laptop just for transferring data is not possible or not affordable now a days when all of us need all devices to be handy.

Moreover, data transmission using laptop or PC involves wastage of lots of power as well as time.

So an effective remedy for this problem is to implement a small and handy device which is able to fulfill our expectations.

\section{BACKGROUND}

Various models have been developed on this theme. But we should pay our attention on overcoming drawbacks of these models.

For achieving this goal we are going use touch screen display instead of keypad, this will reduce the bulkiness and hence device will be more handy.

For achieving higher speed of transmission we are going to use Raspberry Pi.
III.

BLOCK DIAGRAM

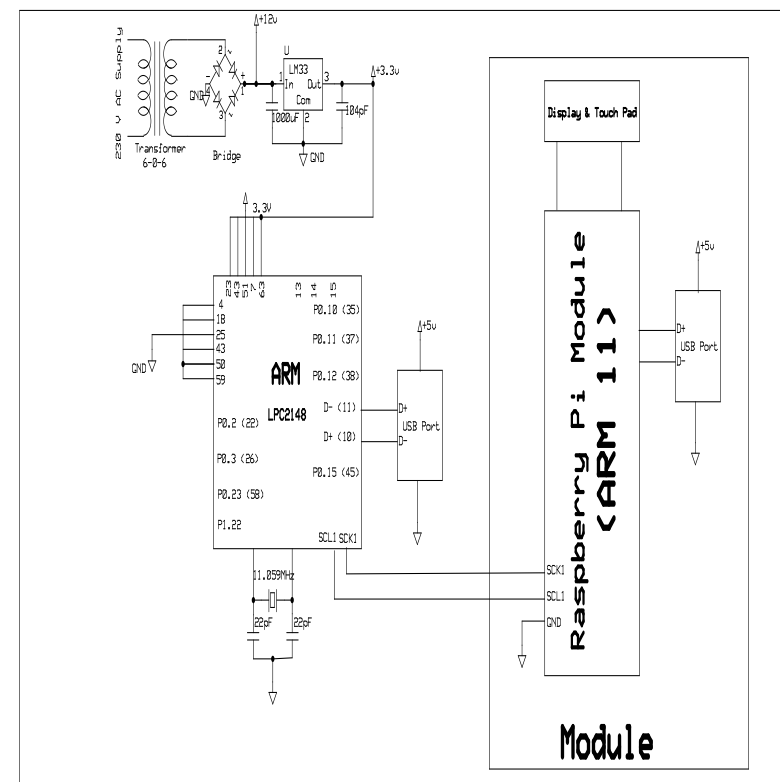

USB to USB Data Transfer Without Computer 
IARJSET

Vol. 4, Special Issue 2, January 2017

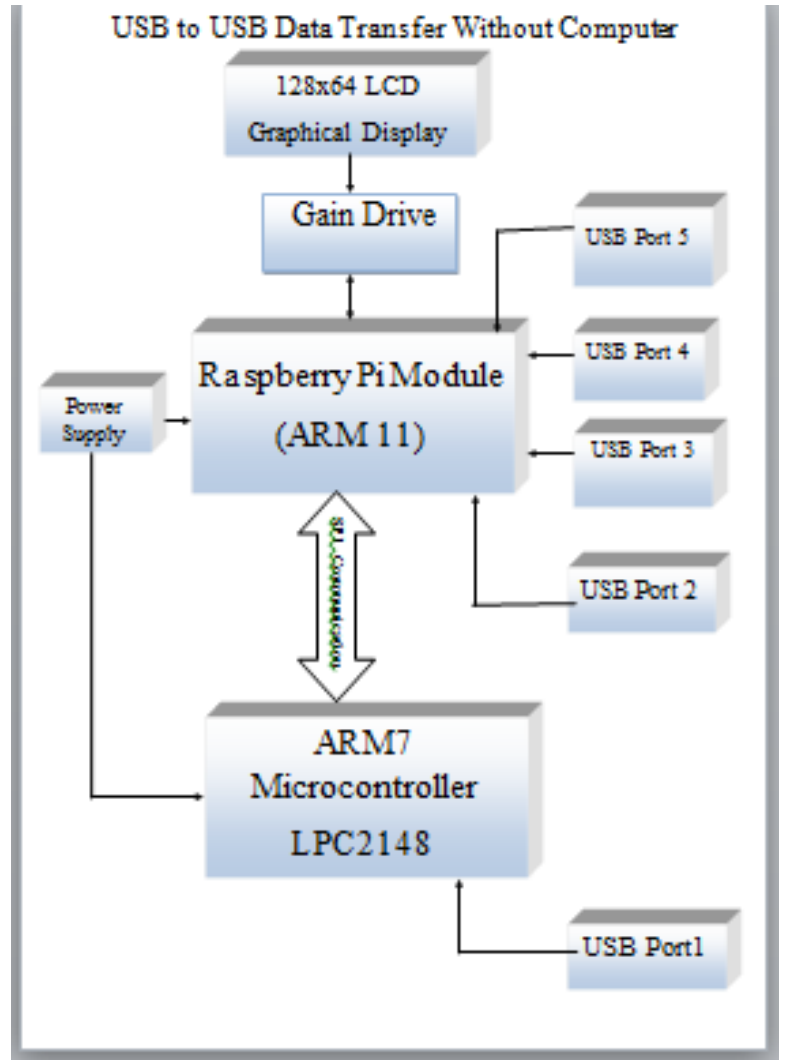

Fig. 2: Block Diagram.

\section{WORKING}

The main intension of our project is to transfer data between USB devices. Raspberry Pi is the heart of our project. One USB is connect to ARM 7 and other four USB's are connected to Raspberry pi. Raspberry pi and ARM7 are connected to one another by means of SPI communication. Graphical display is connected to gain driver and gain driver is connected to Raspberry pi .Gain driver is used to control the display. A USB device which send data is called master and which receive data is called slave. For acknowledgement of insertion of USB devices and data transmission LEDs are used.

\section{HARDWARE REQUIREMENTS}

1. Raspberry PI development board

2. ARM7

3. Graphical Display

4. Light Emitting diode

5. USB Devices

\section{Raspberry Pi:}

Raspberry $\mathrm{Pi}$ is a device as like small computer having size of a credit card. It was first invented in the UK. Raspberry Pi Foundation developed it with. It uses the ARM processor for the hard work in order to operate the Raspberry Pi. ARM processors are very efficient and fast when they are used in small devices. It makes the ARM processor suitable choice for the Raspberry Pi. Even if Raspberry Pi is a computer which does not have a hard drive like general computers, instead it uses SD card for storing the information. SD card does the same job as a hard drive does in a general computer or laptop in Raspberry pi. The SD card contains the operating system, which programs and run the Raspberry $\mathrm{Pi}$. It tells the Raspberry Pi how to work, how to handle any input from the user and how to manage programs when they are running.

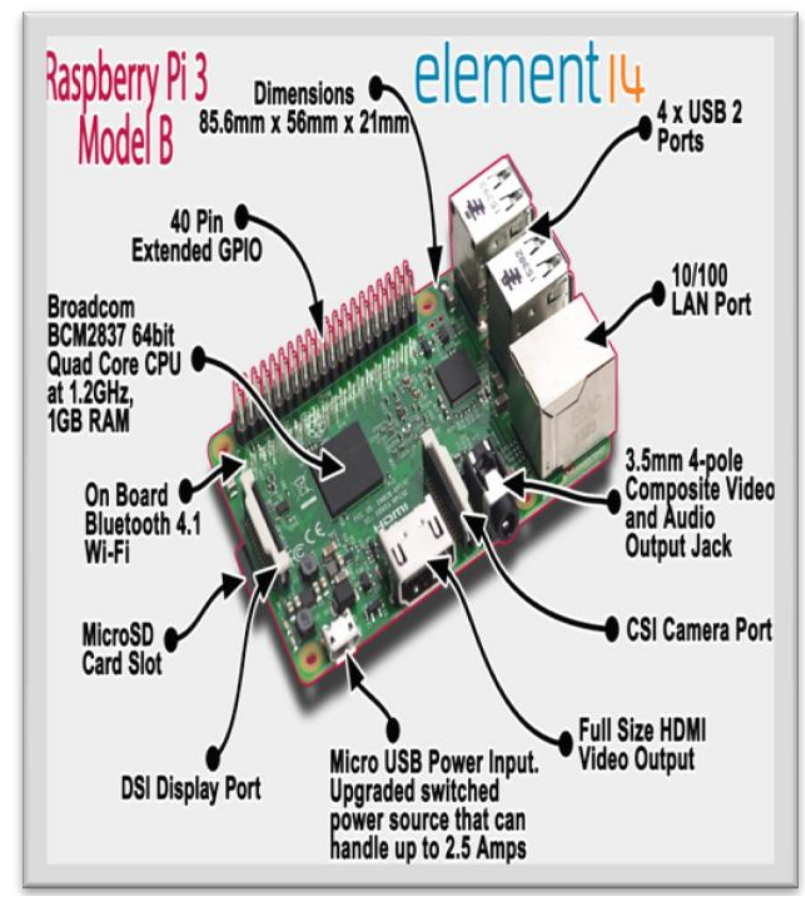

Fig.3:Raspberry PI development board.

\section{ARM7:}

The ARM7 is a general purpose 32-bit microprocessors, which offers low power consumption and high performance. The ARM7 architecture is based on Reduced Instruction Set Computer (RISC), and the instruction set used in it are much simpler than the Complex Instruction Set Computers. Consequently it results in a high instruction throughput and excellent real-time interrupt response from a small chip

For different high-level languages ARM instruction set is a good target for compilers. For critical code segments, assembly code programming is very simple, unlike some RISC processors which depend on refinement compiler technology to manage complicated instruction.

It uses pipelining concept so that all parts of the processing and memory systems can operate continuously. Normally, while one instruction is being executed, its successor is being decoded, and a third instruction is being fetched from memory.

3. Graphical Display :

A display device is an output device for displaying information in visual. It provides users with at a glance data or information. Here we are going to use graphical display which will display the contents of USB devices 
Vol. 4, Special Issue 2, January 2017

connected also the data transmission can be observed using this display.

\section{Advantages:}

1. Here are some advantages of graphical display over other types of displays as discussed below:

2. The cost of graphical display is lower than $\mathrm{KS} 0108$ LCDs

3. The Serial interface of graphical display uses 4 or 5 digital pins.

4. It has low power consumption, full-color RGB LED backlight.

5. It is visible in daylight without backlight.

6. It works precisely with $3 \mathrm{~V}$ logic.

\section{LED :}

LED is an abbreviation for Light Emitting Diode. When current passes through it, it glows.

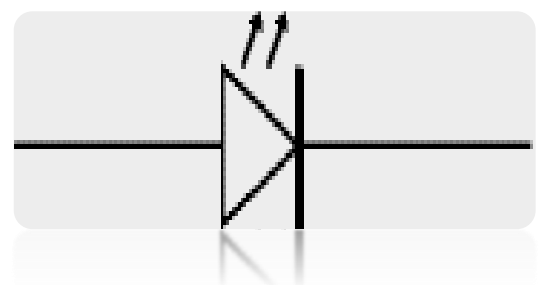

Fig.4: Symbol of LED

There are various colors of LEDs are available in market viz. red, orange, amber, yellow, green, blue and white etc. The color of an LED is determined by the semiconductor material used in it, not by the colorings of the 'package' (the plastic body). Hence in our project we are using such LEDs for getting acknowledgement of how many USB devices are connect as well as the data transmission can be indicated by these LEDs.

\section{USB:}

USB stands for Universal Serial Bus. It was initially developed to standardize the connections of various computer peripherals such as printers, cameras, keyboards, pointing devices etc to PC's to communicate as well as to supply electric power. Generally there exists three basic formats of USB connectors which are: default or standard format intended for PC, mini which is intended for various mobile equipments and last one is thinner which is micro size used for low profile mobile equipments. There exist five modes of data transfer in USB. Those modes are: low speed, full speed, high speed, super speed, super speed+.

\section{SOFTWARE REQUIREMENTS}

\section{Linux:}

Linux is an operating system which is developed for X86 based personal computers. It has been used in computer hardware. It is one of the leading operating systems. It can be installed on PC's, laptops, mobiles and is based on Unix.

2.Python:
Python is not only high level but also object oriented programming language. It is very simple and easy to learn syntax emphasizes readability and therefore reduces the cost of program maintenance.

\section{FUTURE SCOPE}

The following are the further developments that can be done based on the project.

- $\quad$ USB to printer interface.

- It can be embedded into various devices like TV, DVD player, etc.

- Wireless connectivity using Bluetooth.

- Data can be collected on smart phone along with the base stations.

\section{CONCLUSION}

The project undertaken satisfies the needs of the current generation that requires portable means of carrying data transfers. The important thing is data transfer is done without the involvement of PC. It also provides much security as Linux is a much secured Operating System. The advantage of this device is that it is battery as well as power supply operated \& data transfer can be take place at any time.

\section{REFERENCES}

[1] Mr. TusharSawant ,Mr. Sanjay Deshmukh "USB To USB Data Transfer Without PC" National Conference on Research in Electronics Advances \& Collaborative Technologies - 2013.

[2] Mr.V.S.Gawali,Mr.A.M.Agarkar"Pen drive to pen drive and mobile data transfer using ARM" IOSR Journal of Electronics and Communication Engineering (IOSR-JECE).

[3] Ms. Sonal N. Kawale,Prof. ShraddhaMohod"USB To USB And Mobile Data Transfer Using Arm"Electronics and Communication,TGPCET, Nagpur,India. JETIR (ISSN-23495162), Dec 2014 (Volume 1 Issue 7).

[4] Rupali C. Bachalkar, Shrutika D. Durge, Purvaja V. Pote, Sapana V. Ajmire, Prof. Reetesh V. Golhar"Data Transfer between Two Pendrives without PC".Volume 5, Issue 1, January 2015 ISSN: 2277 128X International Journal of Advanced Research in Computer Science and Software Engineering.

[5] www.raspberrypi.org.

[6] www.usb.org.

[7] www.google.com. 